\title{
An instrument for 3D x-ray nano-imaging
}

\author{
M. Holler, ${ }^{\text {a) }}$ J. Raabe, A. Diaz, M. Guizar-Sicairos, C. Quitmann, A. Menzel, and O. Bunk \\ Paul Scherrer Institut, 5232 Villigen PSI, Switzerland
}

(Received 30 May 2012; accepted 29 June 2012; published online 26 July 2012)

\begin{abstract}
We present an instrument dedicated to 3D scanning x-ray microscopy, allowing a sample to be precisely scanned through a beam while the angle of $x$-ray incidence can be changed. The position of the sample is controlled with respect to the beam-defining optics by laser interferometry. The instrument achieves a position stability better than $10 \mathrm{~nm}$ standard deviation. The instrument performance is assessed using scanning x-ray diffraction microscopy and we demonstrate a resolution of $18 \mathrm{~nm}$ in 2D imaging of a lithographic test pattern while the beam was defined by a pinhole of $3 \mu \mathrm{m}$ in diameter. In 3D on a test object of copper interconnects of a microprocessor, a resolution of $53 \mathrm{~nm}$ is achieved. (C) 2012 American Institute of Physics. [http://dx.doi.org/10.1063/1.4737624]
\end{abstract}

\section{INTRODUCTION}

$\mathrm{X}$-ray microscopy provides detailed insight into the structure of matter, a prerequisite for understanding the relation to its function, and has become an important method for imaging in life and materials science samples at the micro and nanoscale. An overview of $\mathrm{x}$-ray microscopy techniques and latest instrumentation can be found in Kaulich et al. ${ }^{1}$ One can separate the field of $x$-ray microscopy in two basic imaging modes: Full-field and scanning microscopy.

In full-field microscopy a resolution below $15 \mathrm{~nm}$ has been demonstrated at soft $\mathrm{x}$-ray energies ${ }^{2}$ by directly imaging the sample onto a 2D detector. By adding a rotational degree of freedom to the sample 3D information can be accessed via tomography, a technique that is nowadays routinely applied and achieves a resolution better than $50 \mathrm{~nm} .{ }^{3-6}$ Several instruments offer cryogenic environments to allow imaging of biological specimens. ${ }^{3-5}$ This is achieved by dedicated developments, but also instrumentation from cryogenic electrontomography ${ }^{7}$ has been successfully adopted to x-ray nanotomography. At hard x-ray energies tomographic full-field imaging is successfully used as well. ${ }^{8,9}$ Recent developments even allow automatic alignment of the individual tomographic projections by measuring the position of the sample on the rotation stage with capacitive sensors. ${ }^{10}$

However, full-field techniques have limitations. At soft $\mathrm{x}$-ray energies, the penetration power of the radiation and the depth of focus are small, which limits the thickness of the sample. At hard x-ray energies the quality of the imaging optics limits the achievable resolution.

Another imaging mode is scanning transmission $\mathrm{x}$-ray microscopy (STXM). ${ }^{1}$ Here, the X-rays are focused to a small spot through which the sample is scanned such that an image is recorded pixel by pixel. Besides a small focal spot, an accurate positioning of the sample in the x-ray beam has to be achieved. Besides a good mechanical arrangement with good stability the measurement of the relative position of the sample to the $\mathrm{x}$-ray focusing optics allowed achieving sample

a) Author to whom correspondence should be addressed. Electronic mail: mirko.holler@psi.ch. positioning with nanometer accuracy. ${ }^{11}$ At soft $\mathrm{x}$-ray energies focal spots of less than $10 \mathrm{~nm}$ in diameter can be generated and an imaging resolution towards $10 \mathrm{~nm}$ has been demonstrated. ${ }^{12}$ But similarly to full-field imaging only thin specimens can be imaged at high resolution due to high absorption and a limited depth of focus.

High energy $x$-rays have an increased penetration and depth of focus, but similarly to full-field imaging the available x-ray optics limit the achievable resolution in hard x-ray STXM. At elevated energies the absorption is significantly reduced which has triggered the development of phase contrast imaging for both full-field and STXM. ${ }^{9,13-15}$ A lensless coherent diffractive imaging (CDI) technique called scanning $\mathrm{x}$-ray diffraction microscopy ${ }^{16,17}$ (SXDM), or ptychography, has shown particular promise, both in terms of resolution ${ }^{18}$ and sensitivity. ${ }^{19}$ By measuring the diffraction intensity patterns at multiple, overlapping sample positions in the coherent x-ray beam, the resolution can be drastically improved and is no longer limited by the transverse spot size of the x-rays. Ptychography retrieves the complex-valued transmissivity of the sample and it neither requires absorption contrast nor does it rely on pure (or weak) phase objects, as Zernike microscopy ${ }^{15,20}$ does, to remain quantitative. The technique is routinely applied by now at various facilities worldwide. ${ }^{18,19,21-26}$

The tomographic application of ptychography is possible by collecting many $2 \mathrm{D}$ projections of specimens at different orientations. ${ }^{27,28}$ By measuring quantitatively the phase advance of $\mathrm{x}$-rays as they traverse the sample, the technique provides with high spatial resolution a quantitative measure of electron density within the sample. ${ }^{29}$

Resolution in tomographic STXM and ptychography is currently often limited by the accuracy and stability of sample positioning in the x-ray beam. High resolution achieved in $2 \mathrm{D}$ scanning microscopy ${ }^{12,18}$ is not trivially extended to $3 \mathrm{D}$ when a rotation of the sample is required. Until now the resolution of ptychographic tomography remained limited to between 100 and $200 \mathrm{~nm}$ due to instabilities and drifts of the more complicated 3D scanning systems, ${ }^{27,30}$ compared to the recently demonstrated resolution of $8 \mathrm{~nm}$ in a $2 \mathrm{D}$ setup. ${ }^{18}$ Here we present a new instrument for high resolution 3D 
scanning x-ray imaging and tomography. We developed and implemented a differential position metrology system, similar to those used in 2D scanning instruments, ${ }^{11}$ but including a rotational degree of freedom in the measurement. In active feedback operation the setup achieves a positioning stability of the sample with respect to the beam-defining optics better than a standard deviation of $\sigma=10 \mathrm{~nm}$. We demonstrate the performance of this instrument using ptychography on 2D and $3 \mathrm{D}$ test samples.

In our demonstration the x-ray beam is defined by a pinhole. To measure a projection, the sample is scanned perpendicular to the direction of beam propagation, and diffraction patterns $\mathrm{I}\left(\mathrm{q}_{\mathrm{x}}, \mathrm{q}_{\mathrm{y}}, \mathrm{x}, \mathrm{y}\right)$ are recorded at each scanning point $(\mathrm{x}, \mathrm{y})$. The obtained reconstructions demonstrate a half period resolution of $18 \mathrm{~nm}$ in a $2 \mathrm{D}$ projection for a lithographic test sample and $53 \mathrm{~nm}$ in 3D on a 3D test sample.

\section{INSTRUMENT COMPONENTS}

\section{A. Mechanical arrangement}

The instrument comprises two independent mechanical subsystems, which are based on commercially available positioning stages. The setup is schematically illustrated in Fig. 1. The following numbers in parentheses refer to the number of the element in this figure. One subsystem positions the $\mathrm{x}$-ray optics, which defines the illumination on the sample, i.e., in this particular case the pinhole (2). The other subsystem is used for sample positioning. These two units are installed on an optical breadboard with dimensions of $1200 \mathrm{~mm}$ $\times 750 \mathrm{~mm}$ such that the entire system can be easily installed at the beamline. In all descriptions the coordinate system is as follows: the $x$-direction points horizontally, $y$ points up perpendicular to the propagation direction of the $\mathrm{x}$-rays, which is $z$.

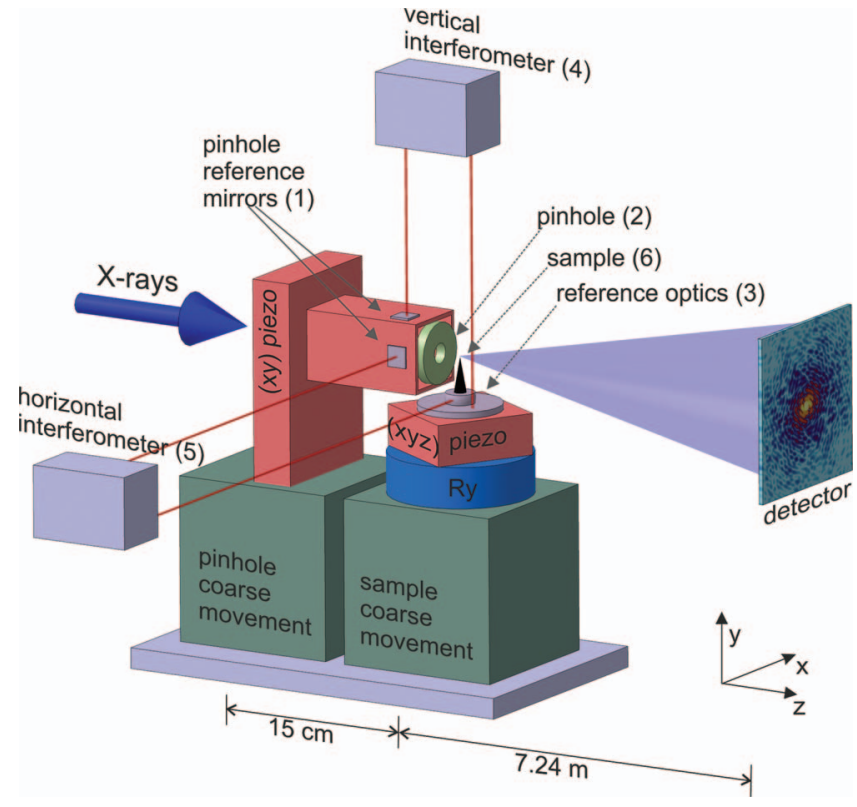

FIG. 1. Schematic of the tomography setup. X-rays enter the setup from the left and propagate through the beam-defining pinhole through the sample to the detector. See text for details.

\section{The pinhole stage}

The pinhole stage consists of a stepper motor driven coarse $x$-stage (Newport, ILS100PP) to which a 2D piezo stage (Physikinstrumente, P-733.2DD) with a travel range of $30 \mu \mathrm{m}(\mathrm{x}, \mathrm{y})$ is attached. The coarse alignment in $y$-direction is realized by moving the table at the beamline, on which the setup is installed. The piezo stage allows fine positioning of the beam-defining optics. The small travel range of this stage results in high stiffness and high resonance frequencies, making the stage suitable for fast position feedback. Here, the beam-defining pinhole was attached to the piezo stage via an extension tube allowing its positioning approximately $3 \mathrm{~mm}$ upstream of the sample.

\section{The sample stage}

The system for positioning the sample consists of two stepper motor driven coarse stages. These allow a sample movement in the $x$-direction (PI Micos GmbH, LS180) and $y$-direction (Newport Inc., UZM160). There is no $z$-motion implemented, neither on the pinhole, nor on the sample stage. For tomography a rotational degree of freedom $\left(\mathrm{R}_{\mathrm{y}}\right)$ is realized using an ultra precision rotation stage (PI Micos GmbH, UPR-160) mounted on top of the two coarse stages. The axis of rotation can be manually aligned to coincide with the $y$ direction. In our configuration a rotation range of $370^{\circ}$ can be covered. For raster scanning of the sample, a three-axes piezo stage (Physikinstrumente, P-563.3CD) is installed on top of the rotation stage. This stage offers a travel range of $300 \mu \mathrm{m}$ in each direction, which is sufficient to center a sample on the axis of rotation and to scan it through the x-ray beam for data acquisition. On the piezo stage the sample mount (6) is installed on top of optical mirrors (3). The latter are required as references for the $x$ - and $y$-position metrology systems $(4,5)$.

\section{B. Metrology system}

To achieve positioning accuracy and stability in the nanometer range one cannot rely on the position encoders built into individual positioning stages. A precise exteroceptive measurement of the relative position of the optical element determining the x-ray illumination (2) with respect to the sample (6) is mandatory. Thus, thermal drifts and parasitic motions can be measured and compensated for. For this it is important to keep the dead-path of the position measurement, i.e., the distance between references and the object of interest, as small as possible.

In the setup presented here, such an exteroceptive metrology is realized by laser interferometry. Laser interferometry offers many advantages: a linear scale, high resolution, long working distance, a large measurement range, and high data-rate. Two interferometers are used, one in $x$-direction (5) and one in $y$-direction (4). A heterodyne helium neon laser is used as light source (Zygo Inc., Model 7714), and the beam is steered by a mirror system to the two interferometers, which are described separately below. The output beams from the interferometers are guided to the analyzing electronics (Zygo Inc., Model 4004) using optical fibers. A measurement 

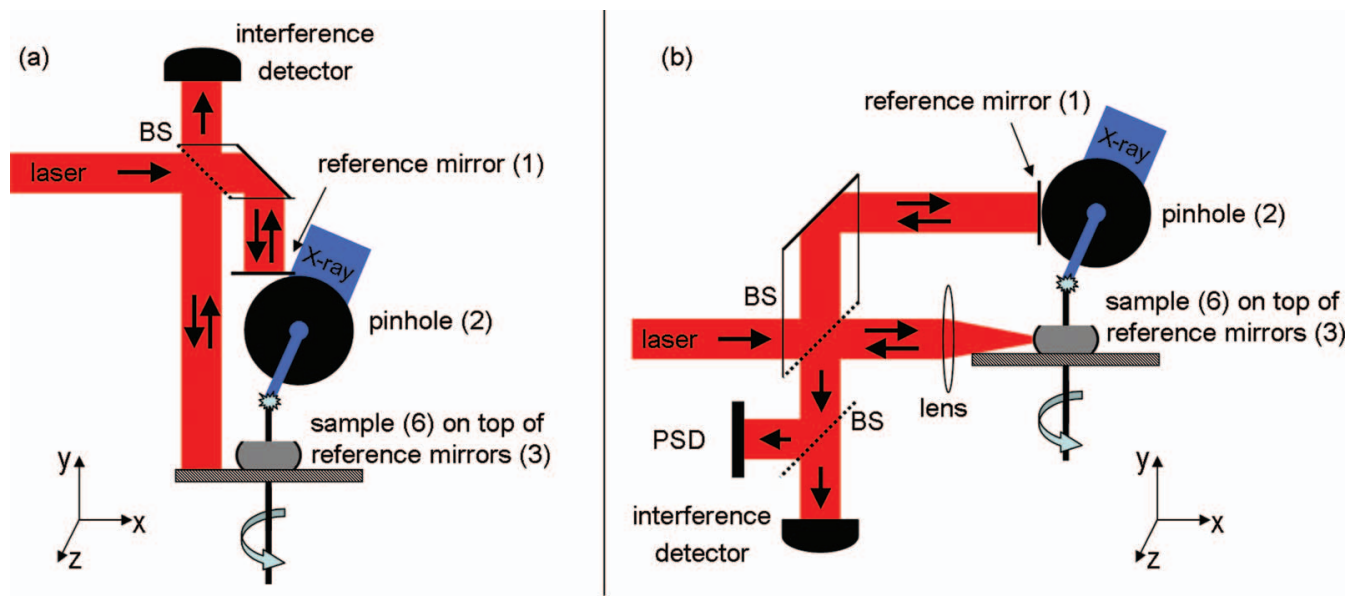

FIG. 2. Schematic illustration of the two laser interferometers used as exteroceptive metrology systems to measure differentially the position of the sample with respect to the illuminating pinhole. (a) Vertical interferometer ( $y$-direction) and (b) interferometer for the horizontal measurement ( $x$-direction), PAT PEND. BS $=$ beam splitter, $\mathrm{PSD}=$ position-sensitive detector.

resolution of up to $0.15 \mathrm{~nm}$ at a noise level below $2 \mathrm{~nm}$ can be achieved at a data rate of tens of kiloHertz.

\section{Vertical interferometer (4)}

For the measurement of the vertical position of the sample (6) with respect to the pinhole (2), a flat mirror aligned perpendicular to the axis of rotation $\left(R_{y}\right)$ is installed on the rotation stage below the sample (3), as shown in Fig. 2(a). At any rotation angle the laser beam of the interferometer is incident on this mirror and reflected back to the interferometer optics. A second flat mirror is installed on top of the pinhole stage to provide the reference, ${ }^{1}$ such that the relative vertical displacement between the sample and pinhole can be accurately measured.

\section{Horizontal interferometer (5)}

To include the rotational degree of freedom in the horizontal interferometer a precision spherical reference mirror (22 $\mathrm{mm}$ diameter, made from tungsten carbide) is implemented between the sample and the flat mirror (3). Its surface roughness is below $10 \mathrm{~nm}$, and its deviation from a perfect sphere was measured to be $\sim 50 \mathrm{~nm}$. To reduce the mobile mass on the sample scanner and the measurement dead-path, only a $6 \mathrm{~mm}$ thick equator disk is installed. To enable an interferometric measurement, the laser beam is imaged onto the sphere as illustrated in Fig. 2(b) using a lens with $100 \mathrm{~mm}$ focal distance, which determines the working distance of the interferometer.

The sample is directly mounted on the reference sphere and will in most cases not be centered on it. For a tomographic measurement, the sample must be centered in the x-ray beam using the piezoscanner. Consequently, the reference sphere will be usually off-center with respect to the rotation axis, which implies that the laser beam reflected from the sphere will not propagate back to the interferometer at all rotation angles. Therefore, the angular beam deflection from the sphere is measured by a position-sensitive detector (PSD) as shown in Fig. 2(b). The signal from the PSD provides a measurement of the position of the sphere in the $y / z$ plane, and the entire interferometer is installed on a $y / z$ translation stage, which can be used to adjust the beam pointing of the interferometer with respect to the sphere. The information from the PSD is used in closed loop to these $y / z$ translation stages. Through this mechanism the pointing of the laser is kept at the center of the sphere for all rotation angles, allowing interferometric position control at all rotation angles without the need to perfectly center a sample on the spherical mirror, which would require additional mechanics between mirror and sample resulting in larger dead-path and thereby in large thermal drifts and reduced stability of the system.

\section{Feedback loop and setup stability}

The signals of the two laser interferometers are used to close a PID (proportional-integral-derivative) feedback loop to the piezo stage of the pinhole. Thereby the relative position of the sample and the pinhole can be actively stabilized. This compensates low frequency vibrations of the system as well as thermal drifts. The feedback loop was operated at $3.3 \mathrm{kHz}$ sampling frequency. The positional stability of the pinhole to the sample which is routinely achieved is $\sigma_{\mathrm{x}}=8 \mathrm{~nm}$ and $\sigma_{\mathrm{y}}$ $=3 \mathrm{~nm}$, should allow achieving high-resolution tomography.

\section{Measurement scheme for the scanning $x$-ray measurement (2D imaging)}

The sample scanner is used to position the sample in the x-ray beam, while the piezo stage of the pinhole is used for closed loop position feedback control. Moving the pinhole is acceptable because the incident illumination of the pinhole is much larger than the pinhole and the amplitude of motion, typically less than $50 \mathrm{~nm}$, is much smaller than the diameter of the pinhole. For x-ray measurements the piezo stage of the pinhole is initially at its center position. The sample is moved to its new position $\left(x=x^{\prime}+\Delta x\right.$, $\left.\mathrm{y}=\mathrm{y}^{\prime}+\Delta \mathrm{y}\right)$ with the sample scanner operated in closed 


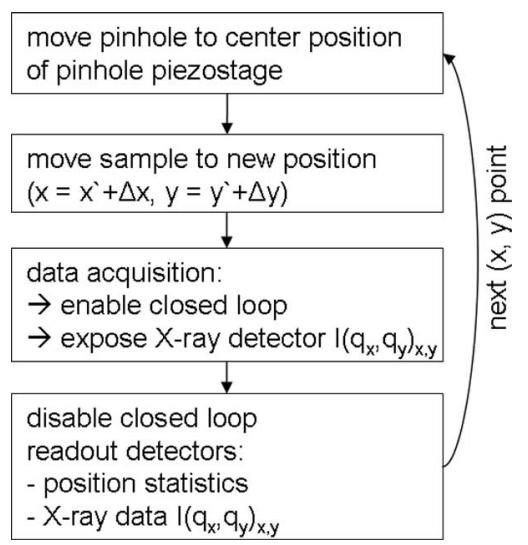

FIG. 3. Schematic of the 2D data acquisition procedure.

loop using internal capacitive position sensors only. At the new position the laser-interferometer closed-loop control is activated synchronized to the start of x-ray data acquisition, such that the relative position of sample and pinhole is stabilized using the piezo stage of the pinhole. While the setup is in closed loop the control system collects the vertical and horizontal position data from the interferometers. After data acquisition the setup with respect to the laser interferometer is put in open loop again, and the pinhole piezo stage is moved back to its center position. The typical amplitude of the motion of the pinhole for position stabilization is less than $50 \mathrm{~nm}$ (peak) in typical acquisition times ranging from $0.5 \mathrm{~s}$ to $2 \mathrm{~s}$. The control scheme is also illustrated in Fig. 3. After switching the feedback system off the mean and standard deviation of the acquired position data from the closed loop operation are calculated. The standard deviation of the positions can be used as diagnostics for the stability of the setup. The mean of the position is directly used as position input for the ptychographic reconstructions.

\section{INSTRUMENT PERFORMANCE}

To demonstrate the performance of the setup, it was installed at the cSAXS beamline (X12SA) of the Swiss Light Source at the Paul Scherrer Institut, Switzerland. For our measurements we used a photon energy of $6.2 \mathrm{keV}$ corresponding to a wavelength of $0.2 \mathrm{~nm}$ and a beam-defining pinhole of $3 \mu \mathrm{m}$ diameter. X-ray diffraction patterns were recorded using a Pilatus $2 \mathrm{M}$ single-photon counting detector ${ }^{31}$ with a pixel size of 172 microns, positioned at a distance of $7.24 \mathrm{~m}$ from the sample. To reduce air scattering and absorption, a Heliumfilled flight tube was installed between the sample and the detector.

\section{A. Results from a 2D test object}

To verify that the positioning stability of the setup indeed allows acquisition of high-resolution tomographic projections, a 2D test pattern was imaged at a single orientation. The sample consisted of a strongly scattering nanofabricated object made of hydrogen silsesquioxane (HSQ). The structure has two concentric circles of HSQ, with diameters of $200 \mathrm{~nm}$ and $600 \mathrm{~nm}$ at two height levels and was subsequently coated with a conformal layer of iridium of about $17 \mathrm{~nm}$ in thickness using atomic layer deposition (ALD), ${ }^{32}$ see the inset in Fig. 5(a).

The sample was measured at 54 points in concentric circles having a radial spacing of $500 \mathrm{~nm}$ covering a field of view (FOV) of $4 \times 4 \mu \mathrm{m}^{2}$. At each point a diffraction pattern was measured with $2 \mathrm{~s}$ exposure time. As in Vila-Comamala et $a l .,{ }^{18}$ the scan was repeated at a different transverse position of the detector in order to measure data that falls in the gaps between detector modules.

The difference map algorithm ${ }^{17}$ was used to reconstruct simultaneously sample and $\mathrm{x}$-ray illumination using an 800 $\times 800$ pixel region of each of the 108 diffraction patterns. This resulted in a real-space pixel size of $10.5 \mathrm{~nm}$. Scanning positions measured by the interferometers were used as input to the reconstruction algorithms, and for each detector position a suitable pixel mask was defined. Thus, 2D measurements from similar areas but not necessarily at exactly the same sample position can be combined. ${ }^{18,33}$

Figure 4(a) shows the reconstructed phase of the test structure. The most prominent features are the Ir-covered side

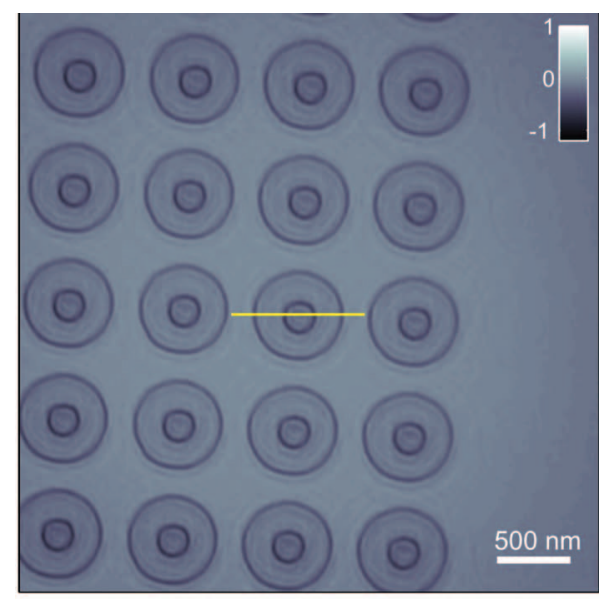

(a)

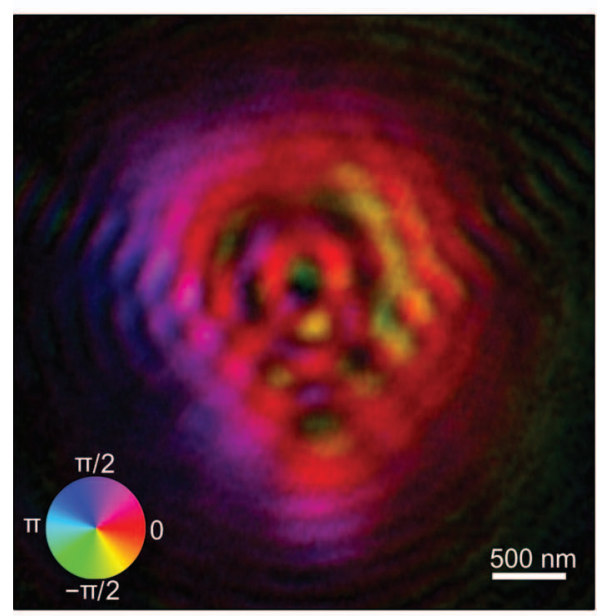

(b)

FIG. 4. (a) Phase of the reconstructed test structures in radians. The solid line indicates the position of the phase profile shown in Fig. 5(a). The radial scanning step of $500 \mathrm{~nm}$ is indicated by the scale bar. (b) Same scale representation of the x-ray illumination on the sample with a size almost as large as the field of view. Amplitude and phase in (b) are shown in linear scale as intensity and hue, respectively. 

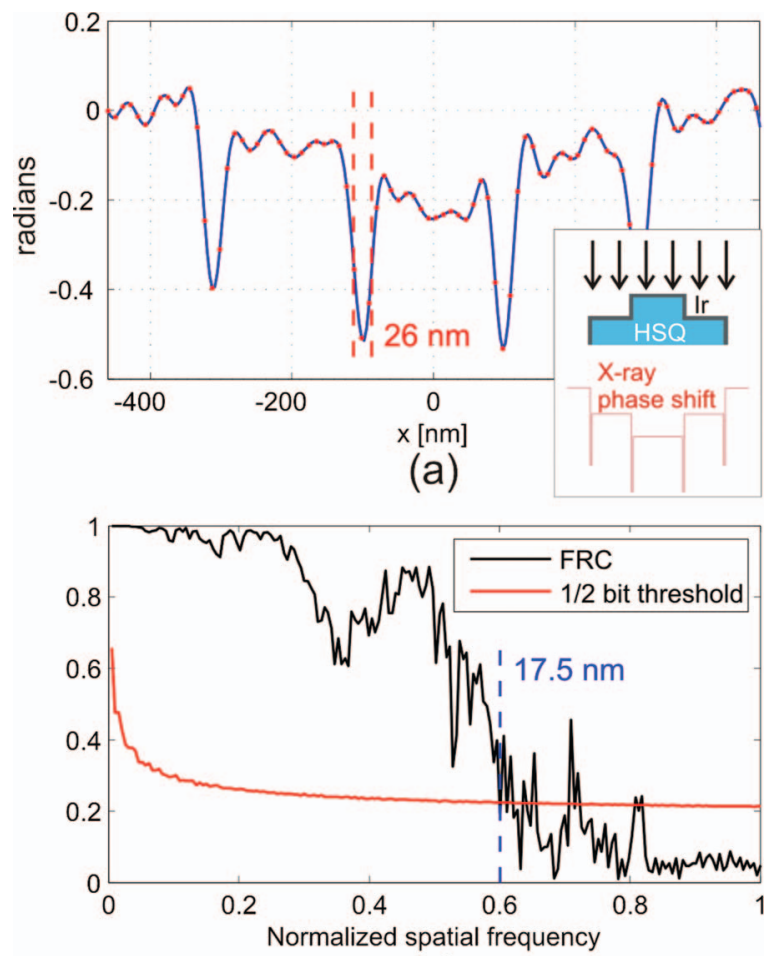

(b)

FIG. 5. (a) Phase profile indicated by the solid line in Fig. 4(a). Measured values are shown as red dots, the blue line is an interpolation. As expected, three phase levels can be observed along with sharp lines originating from the iridium coating. The full width half maximum of $26 \mathrm{~nm}$ on the iridium phase peaks give an upper bound to the resolution. (b) Fourier ring correlation on two independent datasets gives a resolution of about $18 \mathrm{~nm}$.

walls of the HSQ structure. The expected two phase levels, corresponding to the two heights of the HSQ structures, are also reconstructed and better seen in the phase profile shown in Fig. 5(a). The Ir layer is $17 \mathrm{~nm}$ thick but could appear wider due to its high aspect ratio and a possible small misalignment with respect to the direction of $\mathrm{x}$-ray propagation. At the resolution of this image these objects are not small enough to give a direct estimate of resolution. However, an upper limit of the resolution can be obtained by the full width half maximum (FWHM) of the Ir layer peaks giving $\sim 26 \mathrm{~nm}$.

An estimate of resolution independent of having sharp features in the image can be obtained by means of Fourier ring correlation (FRC). ${ }^{34}$ Figure. 5(a) shows the FRC versus spatial frequency of two images acquired under identical conditions. The FRC curve provides a measure of signal-to-noise ratio in Fourier domain and intersects the $1 / 2$ bit threshold ${ }^{34}$ at a resolution of $17.5 \mathrm{~nm}$. Since the FRC decreases monotonically with decreasing SNR in Fourier domain, this first intersection can be interpreted as the highest spatial frequency that presents a SNR of at least 0.42. Figure. 4(b) shows a complexvalued representation of the reconstructed $\mathrm{x}$-ray illumination on the sample with diffraction features arising from free-space propagation across the $3 \mathrm{~mm}$ from the pinhole to the sample. The resolution enhancement obtained by ptychography, defined as the ratio of the probe diameter and the achieved imaging resolution, is in this case 165 .

\section{B. Tomographic imaging}

For the demonstration of nanotomography a sample of copper interconnects obtained from a microprocessor was imaged. One hundred and eighty ptychographic projections were recorded covering an angular range of the incident beam from $0^{\circ}$ to $179^{\circ}$ in steps of $1^{\circ}$. The sample was scanned at 69 points in concentric circles having a radial spacing of $750 \mathrm{~nm}$ and covering a FOV of $10 \times 5 \mu \mathrm{m}^{2}$. At each point a diffraction pattern $\mathrm{I}\left(\mathrm{q}_{\mathrm{x}}, \mathrm{q}_{\mathrm{y}}, \mathrm{x}, \mathrm{y}\right)$ was measured with $1 \mathrm{~s}$ exposure time, and the scan was then repeated at a different transverse position of the detector. Ptychographic reconstructions were performed for each projection, and the resulting phase projections were processed and reconstructed following the procedure described by Guizar-Sicairos et al..$^{28}$ A rendering of the reconstructed structure is shown in Fig. 6. The tomogram reveals several $\mathrm{Cu}$ connections (red) with a thickness of about 1 $\mu \mathrm{m}$ embedded in a $\mathrm{SiO}_{2}$ matrix (grey). The voxel size in this tomogram is $(14 \mathrm{~nm})^{3}$.

In Fig. 7(a), we show a single slice of the tomogram in the $y / z$ plane, revealing several $\mathrm{Cu}$ lines (bright) perfectly aligned to each other within the $\mathrm{SiO}_{2}$ matrix (dark). A horizontal crack of about $200 \mathrm{~nm}$ width introduced in the matrix introduced during sample preparation is perfectly resolved in this image, also recognizable in the $3 \mathrm{D}$ volume representation of Fig. 6 (cf. Video 1). In ptychographic tomography the 3D electron density distribution $n_{\mathrm{e}}(x, y, z)$ is directly obtained. ${ }^{29}$ Assuming that interconnects consist of pure $\mathrm{Cu}$ and the matrix is pure $\mathrm{SiO}_{2}$, we determine a mass density of $(8.50 \pm 0.14)$ $\mathrm{g} / \mathrm{cm}^{3}$ and $(2.31 \pm 0.10) \mathrm{g} / \mathrm{cm}^{3}$, respectively, averaging over volumes of $(300 \mathrm{~nm})^{3}$. While the measured value for silicon dioxide matches the expected density, the expected value for crystalline bulk $\mathrm{Cu}$ of $8.94 \mathrm{~g} / \mathrm{cm}^{3}$ is significantly larger than our measurement, probably due to nanofabrication-related granularity or impurities in this material.

In Fig. 7(b) we show the electron density profile of the object along the red line in Fig. 7(a), revealing the sharp interface between the $\mathrm{Cu}$ and the $\mathrm{SiO}_{2}$ parts of the sample. A detail of one of these interfaces shows a step width of $53 \mathrm{~nm}$. Since such a tomographic slice does not have projection-caused

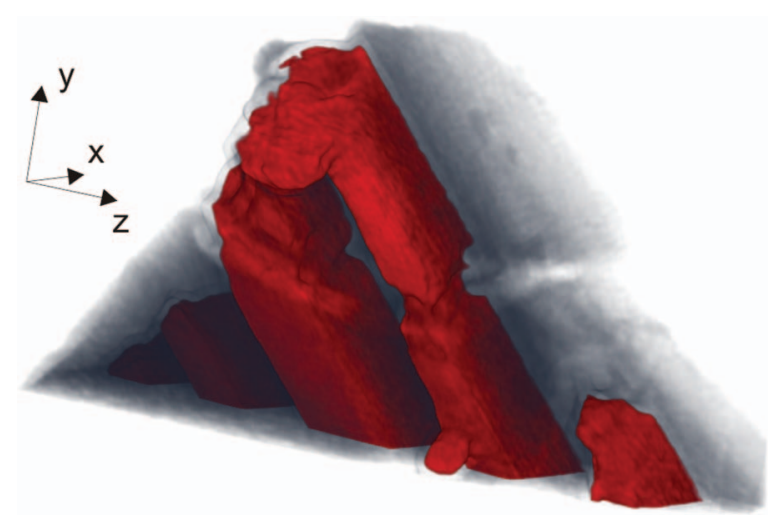

FIG. 6. 3D electron density representation of the phase tomogram of a fragment of a computer processor test sample. We show in red the $\mathrm{Cu}$ connects, while the $\mathrm{SiO}_{2}$ matrix is shown in a semitransparent grey color. The full tomogram has a volume of $9 \times 9 \times 5 \mu \mathrm{m}^{3}$ (enhanced online) [URL: http://dx.doi.org/10.1063/1.4737624.1]. 


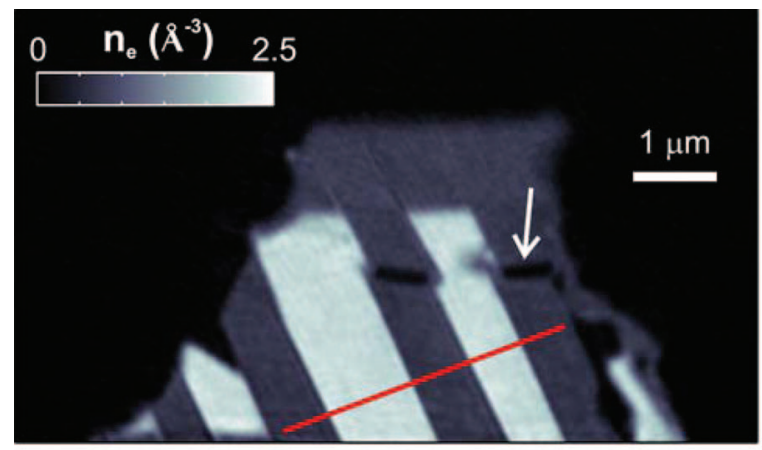

(a)

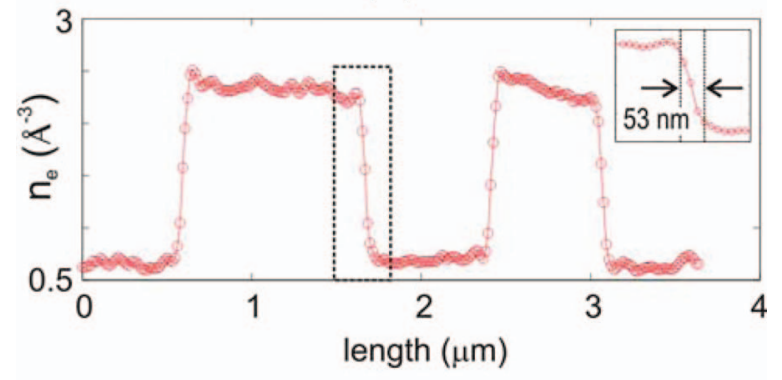

(b)

FIG. 7. (a) $2 \mathrm{D}$ electron density of the tomogram shown in Fig. 6 in the $y / z$ plane. The image reveals the high density contrast between $\mathrm{Cu}$ interconnects and the $\mathrm{SiO}_{2}$ matrix. The arrow indicates a crack in the sample. (b) Profile along the red line in (a). The inset shows a detail of the profile indicated by a dotted square.

feature broadening, this transition can be used as direct estimate of the resolution. To evaluate the resolution of the entire tomogram, we used Fourier shell correlation, which is the analogue in 3D of the FRC described previously. We first computed two independent tomograms with double angular spacing, taking the even and the odd angular steps for each, and we then performed the FSC between the two tomograms. Using the $1 / 2$ bit criterion we find a resolution of $58 \mathrm{~nm}$ for the full 3D tomogram, in good agreement with the line cut shown in Fig. 7(b). To ensure that the resolution estimate is not limited by insufficient angular sampling the resolution was also determined on a smaller region of the tomogram giving the same result.

The resolution in $3 \mathrm{D}$ is worse compared to the resolution achieved in 2D. We attribute this to the weak scattering of the $3 \mathrm{D}$ object compared to the $2 \mathrm{D}$ test pattern but also to instabilities of the X-ray beam that was illuminating the pinhole over the course of the 3D measurement. In our case, the amplitude of beam instabilities from the beamline can be comparable to the diameter of the pinhole. This can become a limitation to reliably image with resolutions below $50 \mathrm{~nm}$, worsening the resolution of some projections and thereby limiting the resolution of the $3 \mathrm{D}$ reconstruction.

\section{SUMMARY AND OUTLOOK}

A setup for scanning x-ray microscopy in 3D has been developed, which offers one rotational degree of freedom for tomography and achieves a position stability of the sample in the $\mathrm{X}$-ray beam of better than $10 \mathrm{~nm}$. The relative position of the beam-defining optics and the sample is measured by an exteroceptive metrology system based on laser interferometry, and the position data are used in an active feedback loop and as input to the image reconstruction algorithm.

The performance of the setup was demonstrated using scanning x-ray diffraction microscopy. The illumination on the sample was defined by a pinhole $3 \mu \mathrm{m}$ in diameter, and a resolution of $18 \mathrm{~nm}$ was obtained in case of a 2D lithographic test sample. In 3D, a resolution of $53 \mathrm{~nm}$ was achieved on a fragment of an integrated circuit. The resolution for the 3D measurement appears currently limited by instabilities of beamline optics causing instabilities of the x-ray beam illuminating the pinhole. Strategies to overcome this are being implemented. Our developments will allow to fully exploit the possibilities of tomographic ptychography.

While in the presented data the alignment of the individual projections of the $3 \mathrm{D}$ dataset was achieved by image processing, ${ }^{28}$ we plan in the future to calibrate the reference mirrors at all rotation angles. Thereby it will become possible to align projections using interferometer position data to allow auto-tomography.

The presented setup is operated in air at room temperature and does not allow cryo-protection of radiation-sensitive samples. In the long term it is planned to implement a similar setup in ultra-high vacuum to allow cooling of samples to liquid-nitrogen temperature and below. It has been demonstrated that imaging sensitivity and dose efficiency is sufficient for unstained biological samples close to their native state. ${ }^{19,21,27}$ Therefore we aim at the measurement of tomograms with resolution down to $10 \mathrm{~nm}$ on biological samples.

\section{ACKNOWLEDGMENTS}

We thank Joan Vila-Comamala and Christian David for providing the $2 \mathrm{D}$ lithographic test object used in the $2 \mathrm{D}$ measurement demonstration. This project is funded by the Competence Centre for Materials Science and Technology (CCMX) of the ETH-Board, Switzerland.

${ }^{1}$ B. Kaulich, P. Thibault, A. Gianoncelli, and M. Kiskinova, J. Phys.: Condens. Matter 23(8), 23 (2011).

${ }^{2}$ W. L. Chao, B. D. Harteneck, J. A. Liddle, E. H. Anderson, and D. T. Attwood, Nature (London) 435(7046), 1210 (2005).

${ }^{3}$ M. A. Le Gros, G. McDermott, and C. A. Larabell, Curr. Opin. Struct. Biol. 15(5), 593 (2005).

${ }^{4}$ G. Schneider, P. Guttmann, S. Heim, S. Rehbein, F. Mueller, K. Nagashima,

J. B. Heymann, W. G. Mueller, and J. G. McNally, Nat. Methods 7(12), 985 (2010).

${ }^{5}$ D. Shapiro, P. Thibault, T. Beetz, V. Elser, M. Howells, C. Jacobsen, J. Kirz, E. Lima, H. Miao, A. M. Neiman, and D. Sayre, Proc. Natl. Acad. Sci. U.S.A. 102(43), 15343 (2005).

${ }^{6}$ Y. Wang, C. Jacobsen, J. Maser, and A. Osanna, J. Microsc. 197, 80 (2000).

${ }^{7}$ A. Leis, B. Rockel, L. Andrees, and W. Baumeister, Trends Biochem. Sci. 34(2), 60 (2009).

${ }^{8}$ J. C. Andrews, S. Brennan, C. Patty, K. Luening, P. Pianetta, E. Almeida, M. C. H. van der Meulen, M. Feser, J. Gelb, J. Rudati, A. Tkachuk, and W. B. Yun, Synchrotron Radiat. News 21(3), 17 (2008).

${ }^{9}$ M. Stampanoni, R. Mokso, F. Marone, J. Vila-Comamala, S. Gorelick, P. Trtik, K. Jefimovs, and C. David, Phys. Rev. B 81(14), 140105 (2010).

${ }^{10}$ J. Wang, Y.-C. K. Chen, Q. Yuan, A. Tkachuk, C. Erdonmez, B. Hornberger, and M. Feser, Appl. Phys. Lett. 100(14), 143107 (2012).

${ }^{11}$ A. L. D. Kilcoyne, T. Tyliszczak, W. F. Steele, S. Fakra, P. Hitchcock, K. Franck, E. Anderson, B. Harteneck, E. G. Rightor, G. E. Mitchell, A. P. Hitchcock, L. Yang, T. Warwick, and H. Ade, J. Synchrotron Radiat. 10, 125 (2003). 
${ }^{12}$ K. Jefimovs, J. Vila-Comamala, T. Pilvi, J. Raabe, M. Ritala, and C. David, Phys. Rev. Lett. 99(26), 264801 (2007).

${ }^{13}$ G. Morrison, W. J. Eaton, R. Barrett, and P. Charalambous, J. Phys. IV France 104, 547 (2003).

${ }^{14}$ P. Thibault, M. Dierolf, C. M. Kewish, A. Menzel, O. Bunk, and F. Pfeiffer, Phys. Rev. A 80(4) 043813 (2009).

${ }^{15}$ U. Neuhausler, G. Schneider, W. Ludwig, M. A. Meyer, E. Zschech, and D. Hambach, J. Phys. D 36(10A), A79 (2003).

${ }^{16}$ J. M. Rodenburg, A. C. Hurst, A. G. Cullis, B. R. Dobson, F. Pfeiffer, O. Bunk, C. David, K. Jefimovs, and I. Johnson, Phys. Rev. Lett. 98(3), 4 (2007).

${ }^{17}$ P. Thibault, M. Dierolf, A. Menzel, O. Bunk, C. David, and F. Pfeiffer, Science 321(5887), 379 (2008).

${ }^{18}$ J. Vila-Comamala, A. Diaz, M. Guizar-Sicairos, A. Mantion, C. M. Kewish, A. Menzel, O. Bunk, and C. David, Opt. Express 19(22), 21333 (2011).

${ }^{19}$ K. Giewekemeyer, P. Thibault, S. Kalbfleisch, A. Beerlink, C. M. Kewish, M. Dierolf, F. Pfeiffer, and T. Salditt, Proc. Natl. Acad. Sci. U.S.A. 107(2), 529 (2010).

${ }^{20}$ H. S. Youn and S.-W. Jung, J. Microsc. 223, 53 (2006).

${ }^{21}$ M. Beckers, T. Senkbeil, T. Gorniak, M. Reese, K. Giewekemeyer, S. C. Gleber, T. Salditt, and A. Rosenhahn, Phys. Rev. Lett. 107(20), 208101 (2011).

${ }^{22}$ C. Rau, U. Wagner, Z. Pesic, and A. De Fanis, Phys. Status Solidi A 208(11), 2522 (2011).

${ }^{23}$ D. J. Vine, G. J. Williams, J. N. Clark, C. T. Putkunz, M. A. Pfeifer, D. Legnini, C. Roehrig, E. Wrobel, E. Huwald, G. van Riessen, B. Abbey,
T. Beetz, J. Irwin, M. Feser, B. Hornberger, I. McNulty, K. A. Nugent, and A. G. Peele, Rev. Sci. Instrum. 83(3), 5 (2012).

${ }^{24}$ Y. Takahashi, A. Suzuki, N. Zettsu, Y. Kohmura, K. Yamauchi, and T. Ishikawa, Appl. Phys. Lett. 99(13), 131905 (2011).

${ }^{25}$ A. Tripathi, J. Mohanty, S. H. Dietze, O. G. Shpyrko, E. Shipton, E. E. Fullerton, S. S. Kim, and I. McNulty, Proc. Natl. Acad. Sci. U.S.A. 108(33), 13393 (2011).

${ }^{26}$ S. Hoenig, R. Hoppe, J. Patommel, A. Schropp, S. Stephan, S. Schoeder, M. Burghammer, and C. G. Schroer, Opt. Exp. 19(17), 16324 (2011).

${ }^{27}$ M. Dierolf, A. Menzel, P. Thibault, P. Schneider, C. M. Kewish, R. Wepf, O. Bunk, and F. Pfeiffer, Nature (London) 467, (7314), 436 (2010).

${ }^{28}$ M. Guizar-Sicairos, A. Diaz, M. Holler, M. S. Lucas, A. Menzel, R. A. Wepf, and O. Bunk, Opt. Exp. 19(22), 21345 (2011).

${ }^{29}$ A. Diaz, P. Trtik, M. Guizar-Sicairos, A. Menzel, P. Thibault, and O. Bunk, Phys. Rev. B 85(2), 020104 (2012).

${ }^{30}$ P. Trtik, A. Diaz, M. Guizar-Sicairos, A. Menzel, and O. Bunk, "Density mapping of hardened cement paste using ptychographic X-ray computed tomography," Cem. Concr. Compos. (to be published).

${ }^{31}$ B. Henrich, A. Bergamaschi, C. Broennimann, R. Dinapoli, E. F. Eikenberry, I. Johnson, M. Kobas, P. Kraft, A. Mozzanica, and B. Schmitt, Nucl. Instrum. Methods Phys. Res. A 607(1), 247 (2009).

${ }^{32}$ J. Vila-Comamala, S. Gorelick, V. A. Guzenko, E. Farm, M. Ritala, and C. David, Nanotechnology 21(28), 285305 (2010).

${ }^{33}$ C. M. Kewish, M. Guizar-Sicairos, C. Liu, J. Qian, B. Shi, C. Benson, A. M. Khounsary, J. Vila-Comamala, O. Bunk, J. R. Fienup, A. T. Macrander, and L. Assoufid, Opt. Exp. 18(22), 23420 (2010).

${ }^{34}$ M. van Heel and M. Schatz, J. Struct. Biol. 151(3), 250 (2005). 